\title{
Damage localization based on modal strain energy index and evidence theory
}

\author{
Jianhua Zhao ${ }^{1,2,}{ }^{*}$, Minghui Shi ${ }^{2}$, Guansheng Yin $^{2}$ and Xiangdong Lian ${ }^{1}$ \\ ${ }^{1}$ Guangxi Communications Investment Group Co., Ltd., Nanning, Guangxi Zhuang Autonomous Region, 530022, China \\ ${ }^{2}$ Department of Engineering Mechanics, Chang'an University, Xi'an, Shaanxi Province, 710064, China
}

\begin{abstract}
A damage localization approach based on modal strain energy index and evidence theory is proposed in this paper. First, the modal strain energy change ratio (MSECR) method and modal strain energy dissipation ratio (MSEDR) method are used to preliminarily detect the damage locations, respectively. Then, the evidence fusion technique is applied to integrate these results and determine the damage locations of structures. To investigate the effectiveness of the proposed method, a numerical example is considered. The numerical results show that the proposed method achieves a better performance in determining the damage locations of structures.
\end{abstract}

\section{Introduction}

Almost all of the engineering structures will inevitably suffer damages during their service life due to different reasons such as material deterioration, environmental corrosion, fatigue, overloads, traffic bumping, and so on. In order to prevent the sudden failure and ensure the safety and reliability of the structures, it is vital to assess the health status of structures periodically and detect the occurrence of structural damage at an early stage [1]. In the past several years, many methods have been developed for structural damage identification. Among them, the vibration-based methods have gained much more attention due to its ease of vibration testing and relatively low-cost implementation [2-5]. The basic idea of these methods is that changes in the physical parameters (mass, damping, stiffness, etc.) will cause measurable changes in the dynamic characteristics (natural frequencies, mode shapes, modal strain energy, etc.). Therefore, the change of structural characteristic parameters can be used to detect the occurrence of damage in the structure.

Modal strain energy (MSE) is defined as the secondorder derivatives of mode shapes, which is much more sensitive to the small change of structural response than natural frequencies and mode shapes [6]. Kumar et al. [7] utilized the changes of MSE before and after the damage to detect the damage of composite sandwich beam, results show that the proposed method is capable of identifying a relatively low extent of damage in the faces and core for several single and multiple damage cases. Seyedpoor and Yazdanpanah [8] developed a static strain energy based index (SSEBI) to determine potentially damaged elements of the structure. In this method, the SSEBI is based on the change of strain energy of a structure subjected to static load cases between the undamaged and damaged states. Vo-Duy et al. [9] employed an approach based on modal strain energy index and improved differential evolution algorithm to detect damage in the laminated composite structures. Dewangan et al. [10] presented two damage indicators based on modal strain energy to detect the damage of Wind Turbine Gearbox (WTG). From the literature described above, it can be concluded that among the damage identification techniques the modal strain energy-based methods show good performance in locating the damage sites, however, it is inevitable to misjudge the damage locations if only a single damage index is used.

In this paper, a damage localization method base on modal strain energy index and evidence fusion technique is presented. First, two damage indices obtained from the modal strain energy change ratio (MSECR) method and the modal strain energy dissipation ratio (MSEDR) method are used to preliminarily determine the damage locations, respectively. Then, the evidence fusion technique is applied to integrate these results and make a final decision result of damage localization. Finally, a numerical example is illustrated to verify the feasibility and effectiveness of the proposed method.

\section{Damage Localization}

\subsection{Modal strain energy change ratio (MSECR)}

The elemental modal strain energy is defined as the product of the elemental stiffness matrix and the second power of the mode shape component, which has been proven much more sensitively to the local damage. For the $j$ th element and the $i$ th mode, the MSE before and after the occurrence of damage can be written by [11]

\footnotetext{
*Corresponding author's e-mail: jhzhao@chd.edu.cn
} 


$$
\begin{gathered}
M S E_{i j}^{u}=\phi_{i}^{T} K_{j} \phi_{i} \\
M S E_{i j}^{d}=\phi_{d i}^{T} K_{j} \phi_{d i}
\end{gathered}
$$

where $M S E_{i j}^{u}$ and $M S E_{i j}^{d}$ denote the undamaged and damaged MSE of the $j$ th element for the $i$ th mode shape; $\phi_{i}$ and $\phi_{d i}$ are the $i$ th mode shapes of the $j$ th element before and after damage, respectively. Since the location and extent of damage in the structure are unknown, the intact elemental stiffness matrix $K_{j}$ is used instead of the damaged one as an approximation in $M_{S E_{i j}^{d}}$. From equations (1)-(2), it is obvious that the change of modal strain energy of the damaged elements are visible than any other undamaged elements. Therefore, it can used to determine the damage locations by comparing with the modal strain energy in the undamaged and damaged cases. The modal strain energy change ratio (MSECR), defined as follows, has been proven to be an effective indicator for damage localization, i.e.

$$
M S E C R_{i j}=\frac{\left|M S E_{i j}^{d}-M S E_{i j}^{u}\right|}{M S E_{i j}^{u}}
$$

where $j$ and $i$ indicate the element number and mode number, respectively.

In general, in order to improve the accuracy of damage identification, several modes are extracted to calculate the MSECR of each element for locating the damaged elements in the structure. For several modes, $M S E C R_{j}$ is defined as the damage localization indicator of the $j$ th element, which is the average of the summation of $M S E C R_{i j}$ for all the modes normalized with respect to the largest value $M S E C R_{i, \max }$ of each mode, can be expressed as

$$
\operatorname{MSECR}_{j}=\frac{1}{m} \sum_{i=1}^{m} \frac{M^{\prime} S E C R_{i j}}{M S E C R_{i, \text { max }}}
$$

where $m$ is the total number of modes. The value of $M S E C R_{j}$ indicates the probability of damage location in the $j$ th element.

\subsection{Modal strain energy dissipation ratio (MSEDR)}

In the viewpoint of energy, the occurrence of structural damage can be described as a process of energy dissipation. Thus, the element-based modal strain energy dissipation ratio can be given by [12]

$$
\varphi_{j}(t)=\frac{-\dot{d}_{j}(t)}{\left[1-d_{j}(t)\right]^{2}} M S E_{j}^{u}
$$

In the initial state, $t=0$ and $d_{j}(t)=0$ represents that the $j$ th element is undamaged. If the damage has occurred, assuming that there is linear relationship between $d_{j}(t)$ and $t$, and then $\dot{d}_{j}(t)$ is a constant.
When $t=t^{d}$, the modal strain energy dissipation of the $j$ th element is calculated as

$$
\int_{0}^{t^{d}} \varphi_{j}(t) d t=M S E_{j}^{u} \int_{0}^{t^{d}} \frac{-\dot{d}_{j}(t)}{\left[1-d_{j}(t)\right]^{2}} d t=M S E_{j}^{u} \int_{0}^{t^{d}} \frac{1}{\left[1-d_{j}(t)\right]^{2}} d\left(d_{j}(t)\right)
$$

According to the law of conservation of energy, the change of MSE before and after the damage occurred should be equal to the strain energy dissipation, so it can be obtained

$$
\left|M S E_{j}^{d}-M S E_{j}^{u}\right|=M S E_{j}^{u}\left|\int_{0}^{t^{d}} \frac{1}{\left[1-d_{j}(t)\right]^{2}} d\left(d_{j}(t)\right)\right|
$$

If the damage index of the $j$ th element at time $t^{d}$ is denoted by a notation $d_{j}$, for which the solution of equation (7) can be written as

$$
d_{j}=\frac{\left|M S E_{j}^{d}-M S E_{j}^{u}\right|}{\left|M S E_{j}^{d}-M S E_{j}^{u}\right|+M S E_{j}^{u}}
$$

It should be pointed out that the damage index $d_{j}$ of damaged elements are significantly large in comparison with the values of undamaged ones.

\subsection{Damage localization using evidence theory}

The evidence theory $[13,14]$, a common tool in data fusion, can aggregate data coming from different information sources and improve the accuracy of final decision-making by providing more complete information. In our study, firstly, we consider the modal strain energies of each element as the initial source of information. Then, two damage indices (MSECR and MSEDR) are constructed by the changes of elemental modal strain energies before and after damage occurred. For the purpose of detecting the potential damage locations preliminarily, the MSECR values and MSEDR values are obtained by equations (4) and (8). Accordingly, the basic identification results can be converted into the local decisions of damage localization. Finally, the local decisions are fused by the evidence theory and a global decision of damage localization can be acquired. The aim of doing this is to improve the accuracy and reliability of damage localization. The details for damage localization using the evidence fusion technique can be described as follows. Assume that there are $N E$ elements need to be identified in the structure and the frame of discernment in evidence theory can be expressed as $A=\left\{A_{1}, A_{2}, \cdots, A_{N E}\right\}$. As mentioned above, the local decisions $m_{1}$ and $m_{2}$ can be obtained by using the MSECR method and MSEDR method, respectively. Then, the MSECR values and MSEDR values will be further processed and acted as BPA values for all the elements, i.e., each of MSECR values and MSEDR values is normalized with respect to the sum of all values. Obviously, they satisfy the conditions of BPA function, i.e., $\sum_{i=1}^{N E} m_{1}\left(A_{i}\right)=1$ and $\sum_{i=1}^{N E} m_{2}\left(A_{i}\right)=1$. As two independent evidence bodies, the BPA $m_{1}$ and $m_{2}$ 
are synthesized by using the Dempster's rule of combination. As a result, we can obtain the final fusion decision results of damage localization, which will provide more accurate results than any individual method.

\section{$3 \quad$ Numerical example}

A 31-bar planar truss structure (shown in figure 1) is employed to verify the effectiveness of the presented method. The basic parameters of materials and geometric properties for the truss structure are given as: elastic modulus $E=70 \mathrm{GPa}$, Poisson's ratio $v=0.3$, mass density $\rho=2.77 \times 10^{3} \mathrm{Kg} / \mathrm{m}^{3}$, the length of each element $L=1.52 \mathrm{~m}$, cross-sectional area $A=0.004 \mathrm{~m}^{2}$. The finite element model of the truss consists of 14 nodes and 31 elements. The total number of degrees of freedom is 25 . Two multiple damage cases are investigated in the example and the structural damage is introduced as a reduction in the stiffness of individual bars. In case A, it is assumed that elements 6 and 13 have damaged with a stiffness reduction of $30 \%$ and $20 \%$, respectively. And in case B, it is supposed that elements 3,14 and 15 have 20\%, $30 \%$ and $15 \%$ reduction in stiffness, respectively. Assuming that the finite element model can accurately simulate the actual dynamic characteristics of the structure, and the measured data are also generated from the finite element model developed in ANSYS environment. Modal analysis is again carried out to obtain the modal parameters in all the situations. Comparison of natural frequencies before and after damage is given in Table 1.

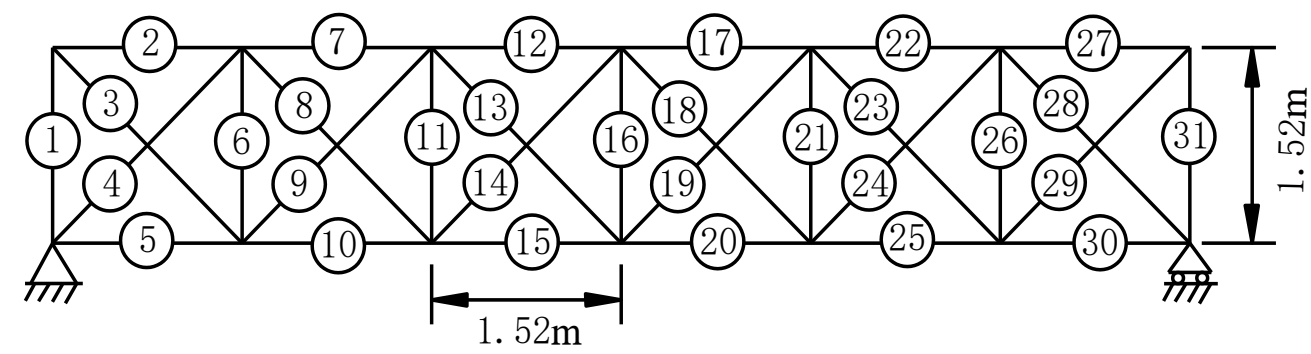

Figure 1. A 31-bar truss structure.

Table 1. Comparison of natural frequencies $(\mathrm{Hz})$ before and after damage.

\begin{tabular}{cccccc}
\hline \multirow{2}{*}{ Mode No. } & \multirow{2}{*}{ Undamaged } & \multicolumn{4}{c}{ Damaged } \\
\cline { 3 - 6 } & & Case A & Error (\%) & Case B & Error (\%) \\
\hline 1 & 36.432 & 36.424 & 0.02 & 35.867 & 1.55 \\
2 & 76.095 & 75.821 & 0.36 & 75.305 & 1.04 \\
3 & 133.81 & 132.82 & 0.74 & 132.56 & 0.93 \\
4 & 223.38 & 222.62 & 0.34 & 221.55 & 0.82 \\
5 & 250.18 & 249.04 & 0.46 & 246.44 & 1.49 \\
\hline
\end{tabular}

For the damage cases $\mathrm{A}$ and $\mathrm{B}$, the results of damage localization using three approaches are plotted against all the element numbers in figures 2 and 3, respectively. In the process of damage identification, the first five modes for damaged and undamaged states are used for detecting the damage locations. As observed in figure 2, it is clear that the damage index values of the two true damaged elements 6 and 13 using the evidence fusion method are significantly greater than those using the MSECR method and MSEDR method. Thus, it is concluded that the damage identification results by evidence fusion method are performing better than that by MSECR method or MSEDR method. Specifically, it can be seen in figure 2 that some high values of damage have also appeared in the neighbourhood of the damaged ones. By comparing figures 2(a)-(c), one notices that the amount of these false detected damage elements is much less obtained by evidence fusion method than those of the other two methods. Apparently, similar observations in the results are made as in figure 3 for damage case B. As can be seen from figures 3(a)-(c), the damage index values of elements 3,14 and 15 are higher than those of other elements. According to this, elements 3, 14 and 15 are judged as the damaged ones. 

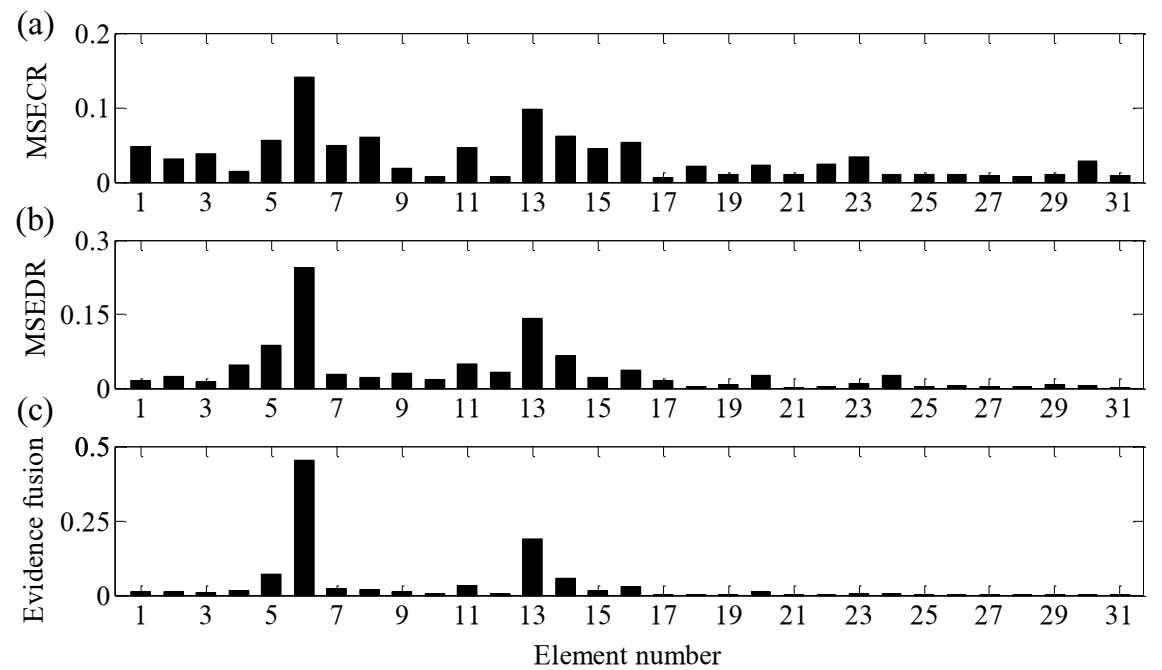

Figure 2. Location of damage for case A using the different methods: (a) MSECR, (b) MSEDR and (c) Evidence fusion.
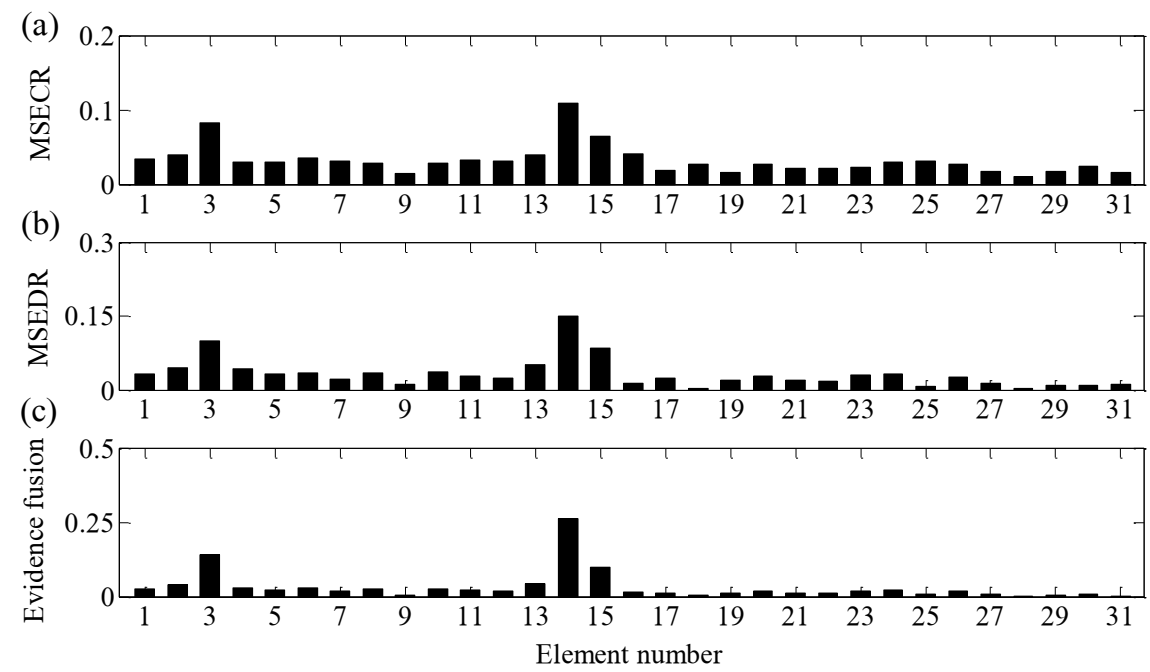

Figure 3. Location of damage for case B using the different methods: (a) MSECR, (b) MSEDR and (c) Evidence fusion.

\section{Conclusions}

In this paper, a damage localization method based on the modal strain energy index and evidence fusion technique is presented to determine the damage location of structures. First, the modal strain energy change ratio (MSECR) method and modal strain energy dissipation ratio (MSEDR) method are employed to preliminarily detect the locations of damage, respectively. Then, the evidence fusion technique is utilized to integrate these results and precisely determine the locations of damage. The effectiveness of the presented method is examined by a numerical example. Simulation results demonstrate that the evidence fusion technique performs much better in determining damage locations than that based on a single method.

\section{Acknowledgments}

This work is supported by China Postdoctoral Science Foundation (2017M623086), Social Development Project of Shaanxi Province (2018SF-360) and Fundamental Research Funds for the Central Universities of China (No. 300102128109).

\section{References}

1. Zhang, D., Johnson, E.A. (2014) Substructure identification for plane frame building structures. Eng. Struct., 60: 276-286.

2. Alvandi, A., Cremona, C. (2006) Assessment of vibration-based damage identification techniques. J. Sound Vib., 292: 179-202.

3. Yan, Y.J., Cheng, L., Wu, Z.Y., Yam, L.H. (2007) Development in vibration-based structural damage 
detection technique. Mech. Syst. Signal Process., 21: 2198-2211.

4. Fan, W., Qiao, P. (2011) Vibration-based damage identification methods: a review and comparative study. Struct. Health Monit., 10: 83-111.

5. Jahangiri, M., Najafgholipour, M.A., Dehghan S.M., Hadianfard, M.A. (2019) The efficiency of a novel identification method for structural damage assessment using the first vibration mode data. J. Sound Vib., 458: 1-16.

6. Hu, H., Wu, C. (2009) Development of scanning damage index for the damage detection of plate structures using modal strain energy method. Mech. Syst. Signal Process., 23: 274-287.

7. Kumar, M., Shenoi, R.A., Cox, S.J. (2009) Experimental validation of modal strain energies based damage identification method for a composite sandwich beam. Compos. Sci. Technol., 69: 16351643.

8. Seyedpoor, S.M., Yazdanpanah, O. (2014) An efficient indicator for structural damage localization using the change of strain energy based on static noisy data. Appl. Math. Model., 38: 2661-2672.

9. Vo-Duy, T., Ho-Huu, V., Dang-Trung, H., NguyenThoi, T. (2016) A two-step approach for damage detection in laminated composite structures using modal strain energy method and an improved differential evolution algorithm. Compos. Struct., 147: 42-53.

10. Dewangan, P., Parey, A., Hammami, A., Chaari, F., Haddar, M. (2020) Damage detection in wind turbine gearbox using modal strain energy. Eng. Fail. Anal., 107: 104228.

11. Shi, Z.Y., Law, S.S., Zhang, L.M. (1998) Structural damage localization from modal strain energy change. J. Sound Vib., 218: 825-844.

12. Guo, H.Y., Li, Z.L. (2011) Two-stage multi-damage detection method based on energy balance equation. J. Nondestruct. Eval., 30: 186-200.

13. Dempster, A.P. (1967) Upper and lower probabilities induced by a multivalued mapping Ann. Math. Statist., 38: 325-339.

14. Shafer, G. (1976) A mathematical theory of evidence (Princeton University Press, Princeton). 